\title{
Non-Maxwellian ion velocity distributions observed using EISCAT
}

Article

Published Version

Lockwood, M., Bromage, B. J. I., Horne, R. B., St-Maurice, J.P., Willis, D. M. and Cowley, S. W. H. (1987) Non-Maxwellian ion velocity distributions observed using EISCAT. Geophysical Research Letters, 14 (2). pp. 111-114. ISSN 0094-8276 doi: https://doi.org/10.1029/GL014i002p00111 Available at https://centaur.reading.ac.uk/38900/

It is advisable to refer to the publisher's version if you intend to cite from the work. See Guidance on citing.

Published version at: http://dx.doi.org/10.1029/GL014i002p00111

To link to this article DOI: http://dx.doi.org/10.1029/GL014i002p00111

Publisher: American Geophysical Union

All outputs in CentAUR are protected by Intellectual Property Rights law, including copyright law. Copyright and IPR is retained by the creators or other copyright holders. Terms and conditions for use of this material are defined in the End User Agreement.

\section{www.reading.ac.uk/centaur}

\section{CentAUR}

Central Archive at the University of Reading 
Reading's research outputs online 
Abstract. Recent observations from the EISCAT incoherent scatter radar have revealed bursts of poleward ion flow in the dayside auroral ionosphere which are consistent with the ionospheric signature of flux transfer events at the magnetopause. These bursts frequently contain ion drifts which exceed the neutral thermal speed and, because the neutral thermospheric wind is incapable of responding sufficiently rapidly, toroidal, non-Maxwellian ion velocity distributions are expected. The EISCAT observations are made with high time resolution (15 seconds) and at a large angle to the geomagnetic field $\left(73.5^{\circ}\right)$, allowing the non-Maxwellian nature of the distribution to be observed remotely for the first time. The observed features are also strongly suggestive of a toroidal distribution: characteristic spectral shape, increased scattered power (both consistent with reduced Landau damping and enhanced electric field fluctuations) and excessively high line-of-sight ion temperatures deduced if a Maxwellian distribution is assumed. These remote sensing observations allow the evolution of the distributions to be observed. They are found to be non-Maxwellian whenever the ion drift exceeds the neutral thermal speed, indicating that such distributions can exist over the time scale of the flow burst events (several minutes).

\section{Introduction}

The first suggestion that large field-perpendicular electric fields could produce nonMaxwellian ion velocity distributions was made by Cole in 1971. By adding ion-neutral collisions, Schunk and walker (1972) showed that stable nonMaxwellian distributions could form for low electric fields and large collision frequencies. In this limit, the departures from a biMaxwellian are small; the perpendicular ion temperature, $T_{\perp}$, being elevated above the parallel value, $T_{I}$. St-Maurice and Schunk modelled the distribution functions for greater electric fields and smaller collision frequencies (which are applicable to the auroral F-region) by making use of a variety of models for the ionneutral collision process (see review by StMaurice and Schunk, 1979). Essentially, it was shown that the bi-Maxwellian is a good approximation if the ion drift (in a frame for which the neutrals are stationary) is smaller than the

1. Rutherford Appleton Laboratory

2. British Antarctic Survey

3. Center for Atmospheric and Space Sciences, Utah State University

4. Blackett Laboratory, Imperial college

Copyright 1987 by the American Geophysical Union.

Paper number $6 \mathrm{~L} 6376$.

$0094-8276 / 87 / 006 \mathrm{~L}-6376 \$ 03.00$ neutral thermal speed. However, for larger drifts the distribution function is predicted to be highly non-Maxwellian and takes the form of a torus in velocity space (symmetric about the magnetic field direction) if the ion collision frequency, $v_{\text {in }}$, is smaller than the ion cyclotron frequency, $\Omega_{i}$. The formation of these toroidal distributions by large ion drifts with respect to the neutral gas, is opposed by ion-ion collisions, ion-neutral reactions and plasma microinstabilities (Ott and Farley, 1975). However, if these competing processes become balanced it is possible for the toroidal distribution to exist in quasi steady state (St-Maurice, 1978).

The presence of non-Maxwellian distributions has important consequences for ion-neutral reaction rates and F-region density and composition (St-Maurice and Schunk, 1979). However, observationally they are very elusive. St-Maurice et al (1976) found non-Maxwellian distributions from over 50 Retarding potential Analyser curves from the $\mathrm{AE}-\mathrm{C}$ satellite when the ion drift was large.

A toroidal distribution function should cause a characteristic spectrum to be observed by incoherent scatter radars. Swift (1975) reported two asymmetric E-region and lower F-region spectra consistent with asymmetric non-Maxwellian distributions due to large drifts when $\nu_{\text {in }}>\Omega_{1}$. However, the spread of velocities within the scattering volume could easily be large enough in the E-region to give the same asymmetry by smearing the spectrum. Predictions of the spectral shape for scattering from toroidal distribution plasma in the F-region $\left(\nu_{i n}<\Omega_{i}\right)$ have been made by Raman et al (1981) and Hubert (1984). The effects have been shown to be greatest for large angles between the radar beam and the magnetic field. The tristatic UHF EISCAT radar has revealed biMaxwellian distributions in the auroral oval with $T_{1}>T_{1}$ (Perraut et al., 1984). L $\phi$ vhaug and Flä (1986) have shown that following substorms both the ion drift and the temperature anisotropy can be large enough $\left(T_{\perp} / T_{\|} \simeq 2\right)$ to imply nonMaxwellian distributions. In this letter we present the first observations of F-region nonMaxwellian distributions from an incoherent scatter radar, allowing a study of their evolution and lifetime.

\section{Observations}

Recently, Todd et al (1986) have reported EISCAT observations of rapid bursts of poleward field-perpendicular flow in the morning sector, auroral ionosphere and have shown them to be consistent with the twin-vortex signature of flux transfer events at the magnetopause. Such events are ideal for driving toroidal distributions in that the ion velocity increases from under 100 $\mathrm{ms}^{-1}$ to about $2 \mathrm{kms}^{-1}$ within 15 seconds. The neutral air will have remained virtually unaccelerated during the period of the burst (about 5 minutes). The observations are made using the monostatic UK Special Programme POLAR (van Eyken et al., 1984) for which the radar beam makes an 
angle $\phi=73.5^{\circ}$ with the geomagnetic field. The data presented are for gates 2-5, centred on altitudes 243, 277, 311 and $346 \mathrm{~km}$, for which signal/noise ratios are high enough to allow 15second resolution analysis of the data and yet for which $v_{i n}<\Omega_{i}$. The data were all recorded on 27 October, 1984, when the onset of a large flow burst event was observed at 06:35:30 UT (Todd et al., 1986).

\section{Signal spectra}

Figure 1 shows the spectra of the received radar pulse, recorded during the integration period 06:35:45 - 06:36:00 UT for range gates 2, 3, 4 and 5. The bulk line-of-sight velocities of the plasma, $v_{T}$, for these 4 gates are 361,817 , 1351 and $1941 \mathrm{~ms}^{-1}$ respectively. These spectra are summed over all 5 frequency channels used by the experiment: spectra for each frequency individually show the same features but have greater noise levels. For gate 2 the spectrum has the normal shape, characteristic of a Maxwellian ion velocity distribution with twin peaks due to scattering from ion acoustic waves propagating towards and away from the radar. For gates 4 and 5 the spectra are exotic in that they have one, central peak with only small shoulders near the frequencies corresponding to the ion acoustic phase velocity. For gate 3 the central peak is smaller and comparable in size to the ion acoustic wave peaks. Spectra of the form shown for gates 4 and 5 in Fig. 1 were observed whenever $V_{T}$ exceeded about $1 \mathrm{kms}^{-1}$ during this burst event.

We have identified two ways in which the spectral shape shown in Fig. 1 can be produced by large ion velocities. The first involves a spatial velocity shear within the scattering volume (or equivalently a large temporal velocity variation within the integration period). Such spectra will be published elsewhere but this effect cannot reproduce the increase in spectral power observed in these observations and will not be discussed any further here. The second mechanism has been modelled by Raman et al (1981) and Hubert (1984) and results from a non-thermal ion velocity distribution function. The formation of the central peak of the scattered signal spectrum arises from the slope of the distribution at low velocities for radar beam directions at large angles to to the magnetic field. If this slope is smaller than that for a Maxwellian distribution, the Landau damping is

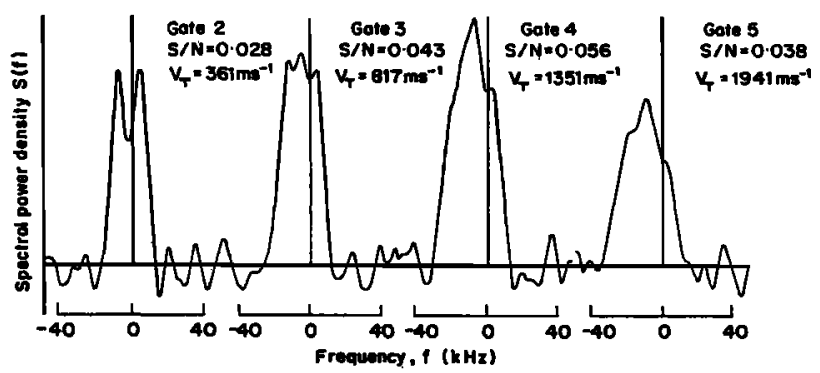

Fig. 1. Signal spectra for the integration period 06:35:45 - 06:36:00 UT on 27 October 1984 for gates 2, 3, 4 and 5 observed by EISCAT using SP-UK-POLAR.

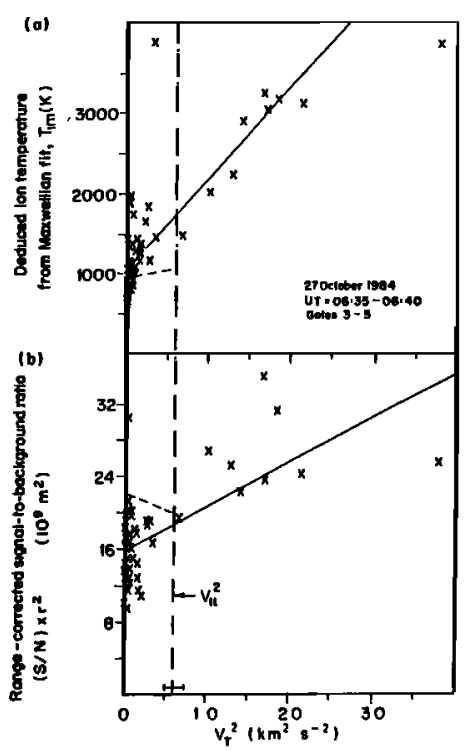

Fig. 2. Scatter plots of (a) the ion temperature deduced from a Maxwellian spectral fit, $T_{i m}$, and (b) the scattered power, $s$, as a ratio of the background noise, $\mathrm{N}$, and corrected to allow for range-squared losses. Plots are as a function of the square of the line-of-sight velocity, $v_{T}{ }^{2}$, and are for the UT period 06:35 - 06:40 and gates 3-5. The dashed lines show the least-squares regression fits for the period 06:30 - 06:35 UT and the same gates.

reduced. At zero phase velocity the dielectric function is smaller and the fluctuation level is greater, giving a large central peak to the scattered spectrum. At the larger, ion-acoustic phase velocity, the distribution slope and hence Landau damping are greater than for a Maxwellian, and hence the normal ion-accoustic wave peaks are suppressed. Note that the departures from a Maxwellian spectrum, predicted by Raman et al (1981), increase with the angle $\phi$ between the radar beam and the geomagnetic field: for small $\phi$ the spectrum is still Maxwellian, but the value of $73.5^{\circ}$ used here is large enough to allow the observation of non-Maxwellian features, yet not so large that coherent echoes from plasma density irregularities are a problem.

\section{Scattered Powers and Inferred Ion Temperatures}

If a scattered signal spectrum for a Maxwellian ion distribution is fitted to that arising from a toroidal distribution, the deduced ion temperature, $\mathrm{T}_{\mathrm{im}}$, will be an overestimate (Raman et al, 1981; Hubert, 1984). The subscript $m$ is used to denote the assumption of a Maxwellian distribution. Values of $T_{i m}$ for the period 06:35 - 06:40 UT (which contains the flow burst) are shown for gates 3, 4 and 5 in Fig. $2 a$ as a function of the square of the line-of-sight velocity, $v_{T}$. Figure $2 b$ shows the observed signal-to-background power ratio, corrected to allow for the range, i.e. $(\mathrm{S} / \mathrm{N}) \times \mathrm{r}^{2}$ which is proportional to the scattered power. It can be seen that both increased sharply with $\mathrm{V}_{\mathrm{T}}{ }^{2}$. The solid lines show regression fits to these data and, by way of comparison, the dashed lines show 
similar regression fits for the period 06:30 06:35 UT, immediately prior to the flow burst when velocity values were low $\left(v_{\mathrm{T}}<300 \mathrm{~ms}^{-1}\right)$. The $V_{T}=0$ intercepts of the fits in Fig. $2 a$ give an estimate of the neutral temperature, $T_{n}$ of $1070 \pm$ 200K. The slope of the fit is $(1.15 \pm 0.24) x$ $10^{-3} \mathrm{~K} \mathrm{~m}^{-2} \mathrm{~s}^{2}$ during the burst period and $10.2 \pm$ $0.8) \times 10^{-3} \mathrm{~K} \mathrm{~m}^{-2} \mathrm{~s}^{2}$ for the five minutes before the event. Theoretically, for a bi-Maxwellian the "line of sight" temperature is given by (Raman et al., 1981; St-Maurice and Schunk, 1979; Perraut et al, 1984):

$T_{i m}=T_{n}+\frac{m_{n}}{2 k_{B}}\left(\beta_{\|} \cos ^{2} \phi+\beta_{\perp} \sin ^{2} \phi\right)\left[\frac{v_{T}}{\cos \alpha}\right]^{2}$

where $k_{B}$ is Boltzman's constant, $m_{n}$ is the mean mass of the neutral gas, $\alpha$ is the angle of the ion velocity vector relative to the line of sight and $\beta_{\|}$and $\beta_{\perp}$ are constants which depend on the collision model. The neutral gas is assumed to be stationary. For $\phi=73.5^{\circ}$ and resonant charge exchange collisions, equation (1) predicts a slope to Fig. $2 a$ of $0.767 \times 10^{-3} / \cos ^{2} \alpha \mathrm{K} \mathrm{m}^{-2} \mathrm{~s}^{2}$ if $\mathrm{m}_{\mathrm{n}}=16 \mathrm{amu}$ (ie atomic oxygen) which lies within the error bar of the slope for the period prior to the burst, suggesting that $m_{n}$ does not exceed 16, ie that there is little molecular gas present. The value observed inside the event is significantly greater than predicted by equation (1), indicating one of four possibilities: (i) a exceeded about $35^{\circ}$; (ii) temporal variations in $\mathbf{V}_{\mathbf{T}}$ within the integration period raised $\boldsymbol{T}_{\mathbf{i m}}$ by smearing the spectrum; (iii) $m_{n}$ exceeded 16amu or (iv) a bi-Maxwellian distribution is inapplicable. Todd et al (1986) have shown $\alpha$ as large as $35^{\circ}$ is very unlikely for this burst event, as is a rapid change in $m_{n}$. We have computed the smearing effect to have caused less than a 48 increase in $T_{i m}$ for the variations of $V_{T}$ observed. However, Raman et al (1981) have shown that toroidal departures from the bi-Maxwellian can cause $T_{i m}$ to be overestimated by the required factor, for the magnitude of ion drift reported here.

St-Maurice and Schunk (1979) have shown that departures from a bi-Maxwellian form are expected if the ion drift velocity, $v_{i}$, exceeds a threshold value, $v_{i t}$, given by:

$$
v_{i t}=0.75 \sqrt{\frac{2 k_{B} T_{n}}{m_{n}}} \text {. }
$$

Using the values for $T_{n}(=1070 \pm 200 \mathrm{~K})$ and $m_{n}$ (= 16amu) deduced above from Fig. 2a, the threshold drift is $V_{i t}=788 \pm 75 \mathrm{~ms}^{-1}$. Hence in Fig. 1 gates 2,3 and 4 represent cases where $v_{T}<$ $v_{i t}, v_{T} \simeq v_{i t}$ and $v_{T}>v_{i t}$ respectively and the central peak appears in a manner fully consistent with the formation of a toroidal distribution. The square of the threshold drift velocity is plotted as the dot-and-dash line in Fig. 2. Figure 2 shows that the signal-to-background ratio, $S / N$, was significantly increased when $V_{T}$ > $v_{i t}$ as was the $T_{i m}$ value.

The plots in Fig. 2 show a great deal of scatter which is to be expected since the data were recorded in two locations roughly $300 \mathrm{~km}$ apart (see Todd et al., 1986) and had contributions from a total altitude range of $243-380 \mathrm{~km}$.

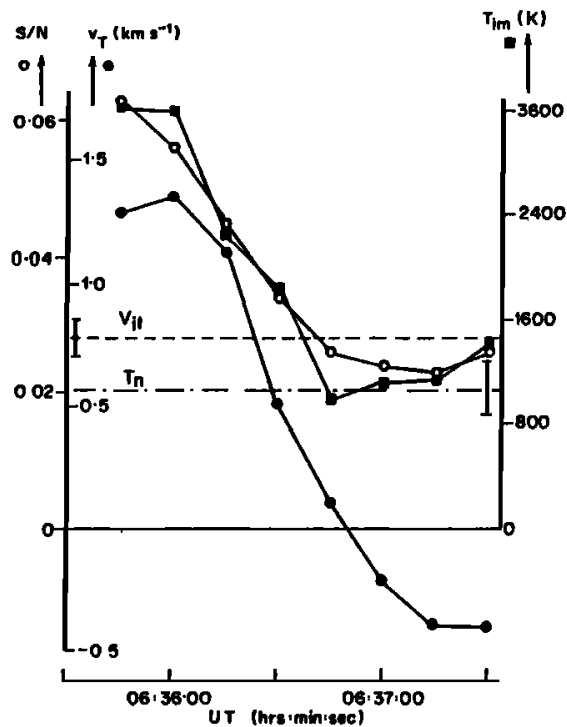

Fig. 3. Variations of the line-of-sight velocity, $V_{T}$, signal to background ratio, $S / N$, and deduced ion temperature assuming a Maxwellian spectrum, $T_{i m}$ • Data are shown for one scattering volume (gate 4). $T_{n}$ is the neutral temperature estimated from figure 2 , and $v_{i t}$ is the threshold ion velocity for driving non-Maxwellian distributions.

Note that a few points in Fig. 2 show high S/N and $T_{i m}$ values, yet low $V_{T}$. Such points may arise from high ion drift and large angle $\alpha$, i.e. there are large drifts perpendicular to the line of sight.

Figure 3 shows the temporal evolution of $T_{1 \mathrm{~m}}$ and $S / N$ in one scattering volume during the flow burst, namely that for gate 4 and radar beam azimuth $356^{\circ}$. The solid circles show the lineof-sight velocity, $V_{T}$, which exceeded the threshold velocity $v_{i t}$ (shown by the horizontal dashed line) for the first three 15-second integration periods. The squares show the ion temperatures (assuming Maxwellian distribution), $T_{i m}$, and the dot-and-dash horizontal line the deduced neutral temperature, $T_{n}$. It can be seen that as $V_{T}$ fell, $T_{i m}$ tended to $T_{n}$, but at the peak of the burst (06:36:00 UT) was roughly 3.5 times larger than $T_{n}$. The received signal-to-background ratio, $S / N$, also fell with $V_{T}$ to a base level of roughly 2.58; however, during the flow burst it was roughly 3 times larger than its value for $V_{T}<$ $v_{i t}$. The higher values of $S / N$ could have been caused by a localised patch of high-density plasmá, however such a patch would be convected around the twin vortex flow burst pattern and hence would not give the simple relationship of $S / N$ to $V_{T}$ shown in Figs. $2 b$ and 3 .

\section{Discussion and Conclusions}

Oux experimental results show that during rapid poleward convection bursts, when the ion velocity exceeds the threshold drift at which a significant departure from a bi-Maxwellian is expected, the incoherently scattered signal spectrum takes on an exotic form of the kind predicted theoretically by Raman et al (1981) and Hubert (1984). The bursts, and hence the 
spectra, are observed only by virtue of the high time resolution of the EISCAT data (15 seconds) and the non-equilibrium effects are observed because the radar beam makes a large angle with the geomagnetic field $\left(73.5^{\circ}\right)$. The rise in scattered signal power is consistent with the effect of the expected non-Maxwellian ion velocity distribution functions and fitting the spectra with a Maxwellian form appears to overestimate the ion temperature by a factor of roughly 2. The shape of the observed spectra reveals that the ion velocity distribution was non-Maxwellian. A full investigation of the form of the distribution function, considering possible effects of noise and signal sampling on the observed spectra, will be presented in a later paper and indicates the distribution function was toroidal, but very close to a flat-topped nonMaxwellian form. It should be noted that if the 'ion acoustic' shoulders on the observed spectra were spurious, the spectra could be explained in terms of coherent echoes from density irregularities driven by plasma instabilities due to the non-Maxwellian plasma (Ott and Farley, 1975). However, at $16.5^{\circ}$ from perpendicularity $\left(\phi=73.5^{\circ}\right)$ such effects would be very small, and it seems therefore unlikely that they can explain the observed rise in scattered power.

These remote observations allow the evolution of the non-Maxwellian distribution function to be observed. Figure 3 shows only the slightest hint of the scattered power returning to its lower, Maxwellian-distribution values (when $v_{T}<v_{i t}$ ) faster than the decay of the ion velocity $V_{T}$, but in general the non-Maxwellian effects are seen whenever the ion velocity exceeds the threshold velocity, $v_{i t}$. Hence the non-Maxwellian distributions at these F-region altitudes have lifetimes of at least the duration of the flow burst events (of order a minute). The slowest mechanism acting to return the distributions to a Maxwellian will be the acceleration of the neutral air which may reduce the relative ionneutral drift to below $v_{i t}$ on the time scale of tens of minutes. The other destructive mechanisms (ion collisions, ion-neutral reactions, microinstabilities) should act on much shorter timescales. Since our observations provide evidence that non-Maxwellian distributions exist for periods of several minutes, they suggest that the destructive mechanisms are balanced in quasi steady state by the driving effect of the ion drift, as postulated by st Maurice (1978).

Acknowledgements. We thank the Director and Staff of EISCAT for their help, S.R. Crothers for graphics software and other staff at RAL for their support and help. EISCAT is supported by the French CNRS, West German MAG, Norwegian NAVF, Swedish NFR, Finnish SA and SERC of the UK.

\section{References}

Cole, K.D., Atmospheric excitation and ionization by ions in strong auroral and man-made electric fields, J. Atmos. Terr. Phys., 33. 1241-1249, 1971 .

van Eyken, A.P., H. Rishbeth, D.M. Willis and S.W.H. Cowley, Initial EISCAT observations of plasma convection at invariant latitudes 7077․ J. Atmos. Terr. Phys., 46, 635-641, 1984. Hubert, D., Non-Maxwellian velocity distribution functions and incoherent scattering of radar waves in the auroral ionosphere, J. Atmos. Terr. Phys., 46, 601-612, 1984.

Lфvhaug, J.P., and T. Flà, Ion temperature anisotropy in the auroral F-region as measured with EISCAT, J. Atmos. Terr. Phys., in press, 1986.

Ott, E., and D.T. Farley, Microinstabilities and the production of short-wavelength irregularities in the auroral $F$ region, J. Geophys. Res., 80, 4599-4602, 1975.

Perraut, S., A. Brekke, M. Baron and D. Hubert, EISCAT measurements of ion temperatures which indicate non-isotropic ion velocity distributions, J. Atmos. Terr. Phys., 46, 531-544, 1984.

Raman, R.S.V., J.-P. St-Maurice and R.S.B. Ong, Incoherent scattering of radar waves in the auroral ionosphere, J. Geophys. Res., 86, 4751-4762, 1981 .

Schunk, R.W., and J.C.G. Walker, Ion velocity distributions in the auroral ionosphere, Planet. Space Sci., 20, 2175-2191, 1972.

St-Maurice, Jo-P., on a mechanism for the formation of VLF electrostatic emissions in the high latitude F-region, Planet. Space Sci., 26, 801-816, 1978.

St-Maurice, J.-P., and R.W. Schunk, Ion velocity distributions in the high-latitude ionosphere, Rev. Geophys, and Space Phys., 17, 99-134, 1979 .

St-Maurice, J.-P.. W.B. Hanson and J.C.G. Walker, Retarding potential analyzer measurement of the effect of ion-neutral collisions on the ion velocity distribution in the auroral ionosphere, J. Geophys. Res., 81, 5438-5446, 1976.

Swift, D.W.. The effect of electric fields and ion-neutral collisions on Thomson scatter spectra, J. Geophys. Res., 80, 4380-4382, 1975.

Todd, H., B.J.I. Bromage, S.W.H. Cowley, M. Iockwood, A.P. van Eyken and D.M. Willis, EISCAT observations of bursts of rapid flow in the high latitude dayside ionosphere, Geophys. Reg. Lett., 13, 909-912, 1986.

B.J.I. Bromage, M. Lockwood and D.M. Willis, Rutherford Appleton Laboratory, Chilton, Didcot, OX11 00X, UK

S.W.H. Cowley, Blackett Laboratory, Imperial College, Iondon, SW7 2BZ, UK

R.B. Horne, British Antarctic Survey, NERC, High Cross, Cambridge, CB3 OET, UK

J.-P. St-Maurice, Center for Atmospheric and Space Sciences, Utah State University, Iogan, Utah 84322-3400, USA

(Received September 9, 1986; revised November 3, 1986; accepted November 14, 1986.) 